\title{
Scattering of inhomogeneous wave by viscoelastic interface crack
}

\author{
P. J. Wei and Z. M. Zhang, Beijing, China \\ (Received November 3, 2000; revised December 15, 2001)
}

\begin{abstract}
Summary. The reflection, refraction and scattering of inhomogeneous plane waves of SH type by an interface crack between two dissimilar viscoelastic bodies are investigated. The singular integral equation method is used to reduce the scattering problem into the Cauchy singular integral equation of first kind by introduction of the crack dislocation density function. Then, the singular integral equation is solved numerically by Kurtz's piecewise continuous function method. The crack opening displacement and dynamic stress intensity factor characterizing the scattered near-field are estimated for various incident angles, frequencies and relaxation times. The differences on crack opening displacement and stress intensity factor between elastic and viscoelastic interface crack are contrasted. And the effects of incident angle, incident frequency and relaxation time of the viscoelastic material are analyzed and explained by the features of phase lag and energy dissipation of the viscoelastic wave.
\end{abstract}

\section{Introduction}

Interaction of waves with an interface crack is an interesting topic and received considerable attention due to the increasing use of composites in engineering. Interface plays an important role in composites as well as in bonded materials. The defects such as micro-cracks and debonded zones on the interface may cause stiffness degradation, and thus influence the integrative properties of composites. Both the detection of interface defects and dynamic failure of the interface are relevant to the interaction of waves with defects. The defects on the interface are generally modeled as interface cracks. Interaction of elastic waves with interface cracks has been studied extensively in the past three decades. For example, Srivastava [1], [2] studied both a two-dimensional interface Griffith crack and three-dimensional interface penny-shaped crack. Yang and Body [3] considered the scattering problem of an interface crack in a layered half-space. Bostrom [4] studied the scattered far field of SH elastic waves. $\mathrm{Qu}$ [5], [6] solved the scattered near-field and far-field of a finite Griffith interface crack in case of an incident plane $P$ wave by using the Fourier transformation and vectorial Cauchy singular integral equation methods.

The waves that propagate in a dissipative medium, for example, viscoelastic materials such as high polymer matrix in composite materials, are different from elastic waves. The natures of attenuation, dispersion, and coupled motion (the trajectory of motion of a particle is elliptical for $\mathrm{P}$ or SV waves) require the introduction of a complex wave number and complex amplitude. The plane wave with complex-valued wave vector and amplitude is generally called inhomogeneous plane wave. Not only the amplitude attenuates in the process of propagation, but also the planes of constant amplitude are not parallel to the planes of constant 
phase. The reflection and refraction of inhomogeneous plane waves were studied firstly by Lockett [7]. It was found that reflection and refraction waves would be fan-shaped sections if incident waves were compound waves with various frequencies. The investigation on the same problem by Cooper [8], [9] has identified the phase shift phenomena between reflection or refraction wave and incident wave. Schoenberg [10] studied the reflection and refraction of an SH wave and the associated energy flow. Borchert [11] studied the reflection and refraction of $\mathrm{P}$ and $\mathrm{S}$ waves and the critical angle of the incident wave. But the investigations on the scattering problem of an inhomogeneous plane wave, in particular, by a crack, were rare to be presented in literature.

In this paper, the scattering problem of an inhomogeneous plane SH wave by an interface crack between two dissimilar viscoelastic bodies is considered. The Fourier transformation and Cauchy singular integral equation method are used. And the numerical results are obtained for the rheology model of a standard linear solid. The crack opening displacement along crack faces and the stress intensity factor at a crack tip are estimated for various incident angles, incident frequency and relaxation time of the viscoelastic material. The procedure in this paper can also be extended to deal with the scattering problems of inhomogeneous plane $\mathrm{P}$ and SV waves which lead to two coupled singular integral equations representing the coupled behavior of stress fields near the interface crack. The details on scattering problems of $\mathrm{P}$ or $\mathrm{SV}$ waves by an interface crack will be provided in another paper.

\section{Statements of the problem}

Consider an interface Griffith crack of length 2a between two dissimilar, homogenous, isotropic, and linearly viscoelastic half-spaces. A Cartesian coordinate system is assumed in such a way that the $\mathrm{x}$-axis is along the crack face and the $\mathrm{y}$-axis is perpendicular to crack faces, as shown in Fig. 1.

The Boltzmann's model of a viscoelastic solid material can be represented through the constitutive equation

$\boldsymbol{\sigma}=\int_{0}^{\infty} \boldsymbol{G}(\tau) \dot{\boldsymbol{\varepsilon}}(t-\tau) d \tau$

where the fourth-order tensor $\boldsymbol{G}(t)$ is called the relaxation function. $\boldsymbol{G}(0)$ is called the instantaneous elastic modulus that governs the instantaneous response to changes in strain, and $\boldsymbol{G}(\infty)$ is called the equilibrium elastic modulus. For an isotropic viscoelastic solid, the relaxation tensor function can be represented by two scalar functions only, i.e. $\mu(t)$ and $\lambda(t)$. The shear relaxation functions of the viscoelastic bimaterials in the problem studied are assumed as $\mu_{1}(t), \mu_{2}(t)$, respectively. And their densities are denoted by $\varrho_{1}, \varrho_{2}$, respectively.

When an oblique incident inhomogeneous plane SH wave travelling from infinity in a viscoelastic solid reaches the interface, reflection, refraction and scattering waves will be generated. The total displacement field can be expressed as

$U=\left\{\begin{array}{ll}U_{1}^{i}+U_{1}^{r}+U_{1}^{s} & y<0 \\ U_{2}^{t}+U_{2}^{s} & y>0\end{array}\right.$,

where $U_{1}^{i}, U_{1}^{r}$ and $U_{2}^{t}$ are the incident, reflection and refraction displacement fields, respectively, for a perfect interface without crack. $U_{1}^{s}$ and $U_{2}^{s}$ are the additional scatter displacement 


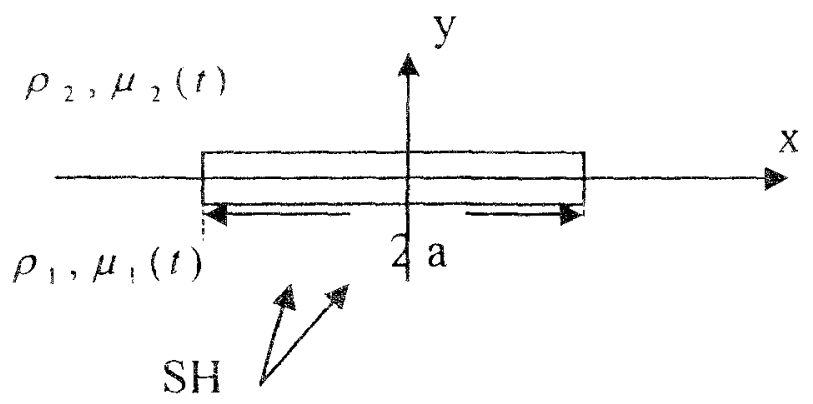

Fig. 1. Inhomogeneous SH plane wave impinging on the interface with crack

fields due to the existence of a crack. The boundary conditions of the problem are given as

$$
\begin{array}{ll}
\sigma_{y z}(x, 0, t)=0 & |x|<a, \\
\sigma_{y z}\left(x, 0^{+}, t\right)=\sigma_{y z}\left(x, 0^{-}, t\right) & -\infty<x<+\infty, \\
U\left(x, 0^{+}, t\right)=U\left(x, 0^{-}, t\right) & |x|>a, \\
U^{s}(x, y, t)=0 & x, y \rightarrow \infty .
\end{array}
$$

\section{Reflection and refraction waves from perfect interface}

In case that the incident wave is an inhomogeneous piane SH wave, no conversion of mode appears, i.e. reflection and refraction waves are inhomogeneous plane SH waves, as shown in Fig. 2. The incident inhomogeneous plane $\mathrm{SH}$ wave can be generally expressed as

$U_{1}^{i}=w_{1}^{i} \exp \left(i \boldsymbol{P}^{i} \cdot \boldsymbol{r}\right) \exp (i \omega t)=w_{1}^{i} \exp i\left(P_{x}^{i} \cdot x+P_{y}^{i} \cdot y\right) \exp (i \omega t)$,

where $\boldsymbol{P}^{i}=\boldsymbol{P}_{R}^{i}+i \boldsymbol{P}_{I}^{i}$ is the complex wave vector with real part $\boldsymbol{P}_{\boldsymbol{R}}^{i}$ and imaginary part $\boldsymbol{P}_{I}^{i}$ being the propagation vector and attenuation vector, respectively. $w_{1}^{i}$ is the complex amplitude in the $z$-direction; $P_{x}$ and $P_{y}$ are the complex projections of complex vector $\boldsymbol{P}^{i}$ in the $x$, $y$-directions, respectively; $\boldsymbol{r}=x \cdot \boldsymbol{x}+y \cdot \boldsymbol{y}$ is the position vector. $\boldsymbol{\omega}$ is the incident frequency. Similarly, the reflection and refraction waves can be expressed respectively as

$$
\begin{aligned}
& U_{1}^{r}=w_{1}^{r} \exp \left(i \boldsymbol{P}^{r} \cdot \boldsymbol{r}\right) \exp (i \omega t)=w_{1}^{r} \exp i\left(P_{x}^{r} \cdot x+P_{y}^{r} \cdot y\right) \exp (i \omega t), \\
& U_{2}^{t}=w_{2}^{t} \exp \left(i \boldsymbol{P}^{i} \cdot \boldsymbol{r}\right) \exp (i \omega t)=w_{1}^{t} \exp i\left(P_{x}^{t} \cdot x+P_{y}^{t} \cdot y\right) \exp (i \omega t)
\end{aligned}
$$

The displacement fields $U_{1}^{i}, U_{1}^{r}, U_{2}^{t}$ satisfy the Helmholtz wave equation with complex wave number

$\nabla^{2} U^{k}+k_{s}^{2} U^{k}=0 \quad$ (superscript $\left.k=i, r, t\right)$,

where $k_{s}=\omega / c_{s}(\omega)$ is the complex-valued wave number; $c_{s}=\sqrt{\mu(\omega) / \varrho}$ is the complex wave speed. $\mu(\omega)=\mu_{0}+\int_{0}^{\infty} \mu^{\prime}(t) e^{i \omega t} d t$ is the complex shear modulus of the viscoelastic material. Inserting the expression of displacement fields $U_{1}^{i}, U_{1}^{r}$ and $U_{2}^{t}$ into Eq. (7) yields

$$
\left.P_{R}^{k} \cdot P_{R}^{k}-P_{I}^{k} \cdot P_{I}^{k}=\operatorname{Re}\left(k_{s 1}^{2}\right) \quad \text { (superscript } k=i, r\right),
$$




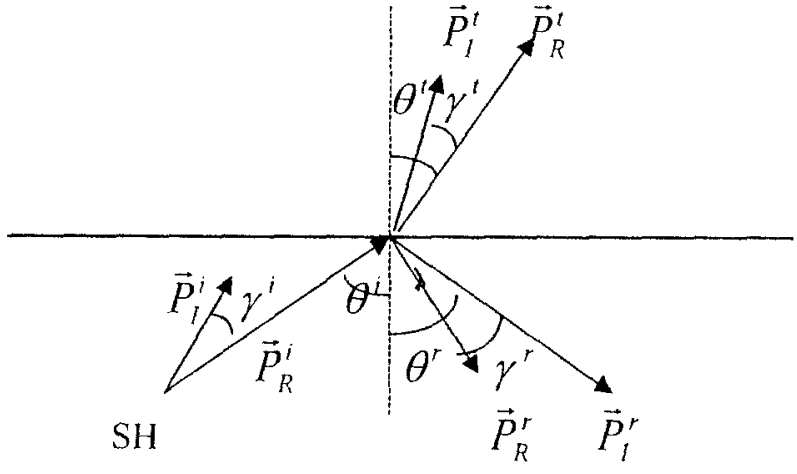

Fig. 2. The reflection and refraction of inhomogeneous SH plane wave on perfect interface.

$\boldsymbol{P}_{\boldsymbol{R}}^{k} \cdot \boldsymbol{P}_{\boldsymbol{I}}^{k}=-\operatorname{Im}\left(k_{s 1}^{2}\right)$,

$\boldsymbol{P}_{\boldsymbol{R}}^{t} \cdot \boldsymbol{P}_{\boldsymbol{R}}^{t}-\boldsymbol{P}_{I}^{t} \cdot \boldsymbol{P}_{I}^{t}=\operatorname{Re}\left(k_{s 2}^{2}\right)$,

$\boldsymbol{P}_{\boldsymbol{R}}^{t} \cdot \boldsymbol{P}_{\boldsymbol{I}}^{t}=-\operatorname{Im}\left(k_{s 2}^{2}\right)$.

Obviously, the propagation vector and the attenuation vector of the incident, reflection and refraction wave can be uniquely determined by material parameters only if the directions of propagation and attenuation of the incident inhomogeneous plane wave are given.

From the continuity of displacement and shear stress across the interface

$U_{1}^{i}+U_{1}^{r}=U_{2}^{t} \quad y=0$,

$\mu_{1}(\omega) \frac{\partial}{\partial y}\left[U_{1}^{i}+U_{1}^{r}\right]=\mu_{2}(\omega) \frac{\partial}{\partial y} U_{2}^{t} \quad y=0$

the reflection and refraction factor and interface shear stress are given by

$\beta_{r}=\frac{w_{1}^{r}}{w_{1}^{i}}=\frac{\mu_{1}(\omega) P_{y}^{i}-\mu_{2}(\omega) P_{y}^{t}}{\mu_{2}(\omega) P_{y}^{t}+\mu_{1}(\omega) P_{y}^{i}}$

$\beta_{t}=\frac{w_{2}^{i}}{w_{1}^{i}}=\frac{2 \mu_{1}(\omega) P_{y}^{i}}{\mu_{2}(\omega) P_{y}^{t}-\mu_{1}(\omega) P_{y}^{i}}$,

$\tau_{y z}(x, 0, \omega)=\mu_{1}(\omega) \frac{\partial}{\partial y}\left(U_{1}^{i}+U_{1}^{r}\right)=\mu_{1}(\omega) w^{i}\left(1-\beta_{r}\right) P_{y}^{i} \cdot \exp \left(i P_{x}^{i} \cdot x\right)$.

It is known that the particle motion of an SH type inhomogeneous plane wave is linear and perpendicular to the plane that propagation and attenuation vector constitute, although it is elliptical for P or SV type inhomogeneous plane waves. Equations (11) and (12) imply that the reflection and refraction waves are $\mathrm{SH}$ type inhomogeneous plane waves only if the incident wave is an SH type inhomogeneous plane wave.

\section{Scattering wave from interface crack}

\subsection{Dual integral equations of crack opening displacement}

The crack opening displacement (COD) is defined as:

$U_{2}^{s}\left(x, 0^{+}, \omega\right)-U_{1}^{s}\left(x, 0^{-}, \omega\right)=\left\{\begin{array}{ll}\Delta w(x, \omega) & |x|<a \\ 0 & |x|>a\end{array}\right.$. 
The scattered displacement field $U^{s}(x, y)$ satisfies the Helmholtz wave equation (7) and radiation condition (3.4). By using the Fourier integral transformation, the scattered displacement fields can be obtained in the transformation domain, i.e. $\tilde{U}^{s}(s, y)$. Then, employing the displacement and stress boundary conditions, i.e. (3.1) and (3.3), leads to the dual integral equations in terms of the crack opening displacement

$$
\begin{cases}F^{-1}[\Delta \tilde{w}]=0 & |x|>a \\ F^{-1}\left[\mu_{1}(\omega) \frac{\beta}{\beta+1} \sqrt{k_{s 1}^{2}-s^{2}} \Delta \tilde{w}\right]=-\tau_{y z}(x, 0, \omega) & |x|<a\end{cases}
$$

where $\beta(s, \omega)=\mu_{2}(\omega) \sqrt{k_{s 2}^{2}-s^{2}} / \mu_{1}(\omega) \sqrt{k_{s 1}^{2}-s^{2}}, \Delta \tilde{w}(s, \omega)=F[\Delta w(x, \omega)]$, and $F[]$ and $F^{-1}[]$ denote the Fourier transformation and inverse Fourier transformation, respectively.

\subsection{Singular integral equation of crack dislocation density}

The crack dislocation density function is defined as

$$
\Phi(x, \omega)=\frac{\partial}{\partial x} \Delta w(x, \omega)
$$

Applying the Fourier integral transformation on $x$ in Eq. (16) yields

$\tilde{\Phi}(s, \omega)=(i s) \Delta \tilde{w}(s, \omega)$.

Then, substituting Eq. (17) into Eq. (15) yields

$$
\begin{aligned}
& \int_{-a}^{a} \Phi(x, \omega) d x=0, \\
& -\frac{1}{2 \pi i} \int_{-\infty}^{\infty} \mu_{1}(\omega) \frac{\beta}{\beta+1} \sqrt{k_{s 1}^{2}-s^{2}} \frac{1}{s}\left[\int_{-a}^{a} \Phi(u) e^{i s u} d u\right] e^{-i s x} d s=-\tau_{y z}(x, 0, \omega) \quad|x|<a .
\end{aligned}
$$

Considering

$\lim _{s \rightarrow \infty} \mu_{1}(\omega) \frac{\beta}{\beta+1} \sqrt{k_{s 1}^{2}-s^{2}} \frac{1}{s}=\frac{\mu_{1}(\omega) \mu_{2}(\omega)}{\mu_{1}(\omega)+\mu_{2}(\omega)} \operatorname{sign}(s)$

and

$$
\int_{-\infty}^{\infty} e^{i s(u-x)} \operatorname{sign}(s) d s=\frac{2 i}{u-x},
$$

Equation (19) is reduced into the Cauchy singular integral equation of first kind

$$
\frac{\mu_{1}(\omega) \mu_{2}(\omega)}{\mu_{1}(\omega)+\mu_{2}(\omega)} \frac{1}{\pi} \int_{-a}^{a} \frac{\Phi(u, \omega)}{u-x} d u+\int_{-a}^{a} K(u, \omega) \Phi(u, \omega) d u=-\tau_{y z}(x, 0, \omega) \quad|x|<\alpha,
$$

where the known integral kernel is represented as

$$
\widetilde{K}(u, \omega)=-\frac{1}{2 \pi i} \int_{-\infty}^{\infty}\left[\mu_{1}(\omega) \frac{\beta}{\beta+1} \sqrt{k_{s 1}^{2}-s^{2}} \frac{1}{s}-\frac{\mu_{1}(\omega) \mu_{2}(\omega)}{\mu_{1}(\omega)+\mu_{2}(\omega)} \operatorname{sign}(s)\right] e^{-i s(u-x)} d s .
$$

The right-hand side of Eq. (21) is obtained from Eq. (13). Thus, the unknown function of the Cauchy singular integral equation (21) is the crack dislocation density function, i.e. $\Phi(u, \omega)$. 


\subsection{Numerical solution of the singular integral equation}

The singular integral equation has been extensively studied in [12]. The conventional method of solving a singular integral equation is based on the regularization of the singular operator through the application of another singular operator (usually the adjoint of the original singular operator). By introduction of the fundamental solution of the singular integral equation (21)

$W(x)=(a-x)^{-1 / 2}(a+x)^{-1 / 2}$

the unknown function in Eq. (21) can be expressed as

$\Phi(x, \omega)=\varphi(x, \omega) W(x)$.

This method leads to a Fredholm integral equation in terms of a new unknown function $\varphi(x, \omega)$ which is bounded and continuous in the interval $|x|<a$. The fundamental solution of the singular integral equation preserves the correct nature of the singularity of the dislocation density function at the crack-tip. The real singular index implies that there is no oscillation singularity for interface cracks under anti-plane shear deformation.

Because of the complexity of the integral kernel $K(u, \omega)$, Eq. (21) can only be solved numerically. There are mainly two numerical methods: one developed by Erdogan [13] is based on the Jacobi or Chebyshev orthogonal polynomial; another developed by Kurtz [14] is based on the piecewise continuous functions. The first method decomposes the unknown function into a series of the form

$\varphi(x, \omega)=\sum_{1}^{N} a_{n}(\omega) P_{n}^{(\alpha, \beta)}(x)=\sum_{1}^{N} a_{n}(\omega) T_{n}(x) \quad\left(\right.$ when $\left.\alpha=\beta=\frac{1}{2}\right)$

and reduces the singular integral equation in terms of the unknown function $\varphi(x, \omega)$ into the algebraic equations in terms of the unknown coefficients $a_{n}(\omega)$. The second method decomposes the unknown function into a continuous function of low order in every subinterval,

$\varphi(x, \omega)=\sum_{1}^{N} \varphi_{i}(x, \omega)$

where $\varphi_{i}(x, \omega)=\sum_{j=1}^{n} \lambda_{i j}(x) \varphi_{i j}(\omega)$ is an interpolation polynomial of $n-1$ order in the subinterval $\left[x_{i}, x_{i+1}\right]$, and reduces the singular integral equation in terms of the unknown function $\varphi(x, \omega)$ into the algebraic equations in terms of the unknown variables $\varphi_{i j}(\omega)$ which are the values of $\varphi(x, \omega)$ at the $j$-th collection point in $i$-th subinterval.

In this paper, the piecewise continuous function method is used for its convenience to evaluate the value of $\varphi(x, \omega)$ at points $x= \pm a$, and $\lambda_{i j}(x)$ adopts the continuous function of second order. The details of the method are not repeated again here. After the crack dislocation density $\Phi(x, \omega)$ is obtained, the crack opening displacement along the crack face and the stress intensity factor at crack-tip can be estimated as follows:

$$
\begin{aligned}
& \Delta w(x, \omega)=\int_{-a}^{x} \Phi(x, \omega) d x=\int_{-a}^{x} \varphi(x, \omega) W(x) d x \\
& \tau_{y z}(x, 0, \omega)=-\frac{\mu_{1}(\omega) \mu_{2}(\omega)}{\mu_{1}(\omega)+\mu_{2}(\omega)} \frac{1}{\pi} \int_{-a}^{a} \frac{\Phi(u, \omega)}{u-x} d u+\int_{-a}^{a} K(u, \omega) \Phi(u, \omega) d u \quad|x| \geq a, \\
& K_{\mathrm{III}}=\lim _{x \rightarrow a} \sqrt{2 \pi(x-a)} \tau_{y z}(x, 0, \omega) .
\end{aligned}
$$



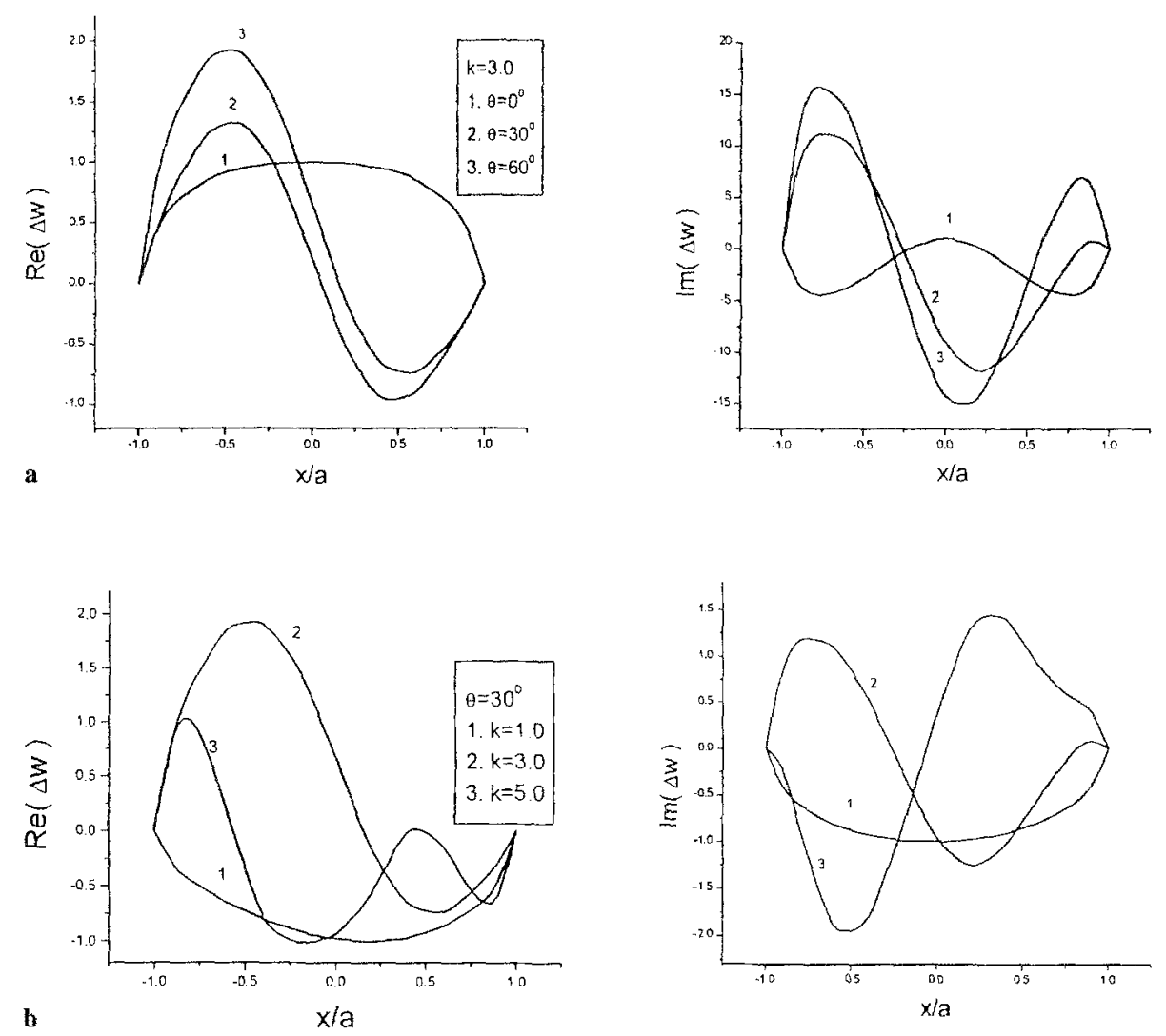

Fig. 3. Crack open displacement along crack face $\Delta w(x)$ for relaxation time $\tau=\infty$

\section{Numerical results and discussion}

The rheologic model describing the dissipative properties of a viscoelastic solid can be represented in terms of various configurations of springs and dashpots. It generally leads to various kinds of differential constitutive equations. Among them, the standard linear solid model is well-known and used in the numerical example. On the other hand, the Boltzmann's model of a dissipative medium based on fading memory generally leads to an integral constitutive equation. The corresponding relaxation function of the standard linear solid model can be expressed as

$\mu_{i}(t)=\mu_{\infty i}\left[1+f_{i} \exp \left(-\frac{t}{\tau_{i}}\right)\right] \quad(i=1,2)$,

where $f_{i}=\mu_{0 i} / \mu_{\infty i}-1$, and $\tau_{i}$ is the relaxation time of the viscoelastic materials.

The stress intensity factor (SIF) indicates the intensity of the scattered stress field at the crack-tip. And the crack open displacement (COD) indicates the magnitude of the scattered displacement field at the crack faces. Both of SIF and COD are computed in this numerical example. Obviously, the crack opening displacement $\Delta w$ and the stress intensity factor $K_{\text {III }}$ are both dependent upon not only the incident wave parameters, i.e. incident angle $\theta^{i}$ and incident frequency $\omega$ or wave number $k$, but also on the viscoelastic material parameters, i.e. relaxation time $\tau$ and modulus difference $f$. In order to show the effects of these parameters, 

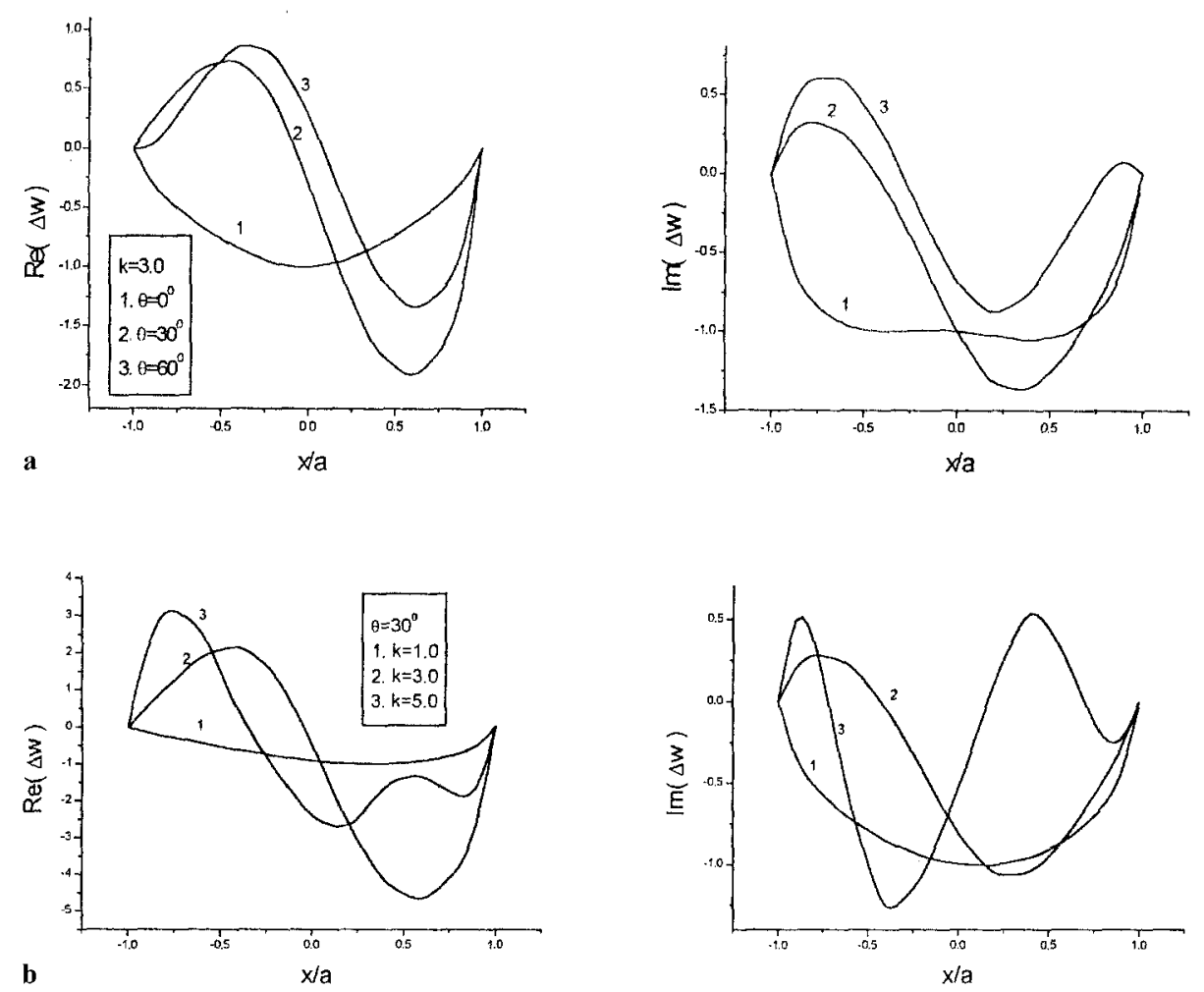

Fig. 4. Crack open displacement along crack face $\Delta w(x)$ for relaxation time $\tau=1 \mathrm{~s}$

Table 1.

\begin{tabular}{lllll}
\hline & $\mu_{0}$ & $\mu_{\infty}$ & $\tau$ & $\varrho$ \\
\hline Material 1 & $1268.0\left(\mathrm{MNm}^{-2}\right)$ & $634.0\left(\mathrm{MNm}^{-2}\right)$ & $0.1(\mathrm{~s})$ & $1200\left(\mathrm{kgm}^{-3}\right)$ \\
Material 2 & $1060.0\left(\mathrm{MNm}^{-2}\right)$ & $264.0\left(\mathrm{MNm}^{-2}\right)$ & $0.1(\mathrm{~s})$ & $1200\left(\mathrm{kgm}^{-3}\right)$ \\
\hline
\end{tabular}

numerical calculations are carried out for various $\theta^{i}, k$ and $\tau$. Other viscoelastic material constants used in the numerical example are listed in Table 1 . And the angle between propagation and attenuation vector of the incident wave is assumed $\gamma^{i}=20^{\circ}$.

The crack opening displacement $\Delta w$ along the crack face for various incident angles $\theta^{i}$ and wave number $k$ are shown in Fig. 3 and Fig. 4. The numerical results in the figures have been normalized by the crack open displacement at the center of the crack. The case that the relaxation time approaches infinity, i.e. $\tau=\tau_{1}=\tau_{2}=\infty$, implies that the interface crack is located between two dissimilar elastic materials. Thus, the numerical results shown in Fig. 3 correspond to an elastic interface crack. In Fig. 4, the case that relaxation time is finite, i.e. $\tau=\tau_{1}=\tau_{2}=1 \mathrm{~s}$, is considered. The case implies that the interface crack is located between two dissimilar viscoelastic materials. Thus the numerical results shown in Fig. 4 correspond to a viscoelastic interface crack. By comparing Fig. 4 with Fig. 3, the effects of viscosity of the material on the scattered displacement field can be seen clearly.

In Fig. 5, the stress intensity factor $K_{\text {III }}$ of scattered stress fields around the crack tip, i.e. $x=a$, is shown for various wave numbers $k$ in the case that the incident angle $\theta^{i}$ is equal to $60^{\circ}$. The numerical results corresponding to an elastic interface crack and viscoelastic inter- 


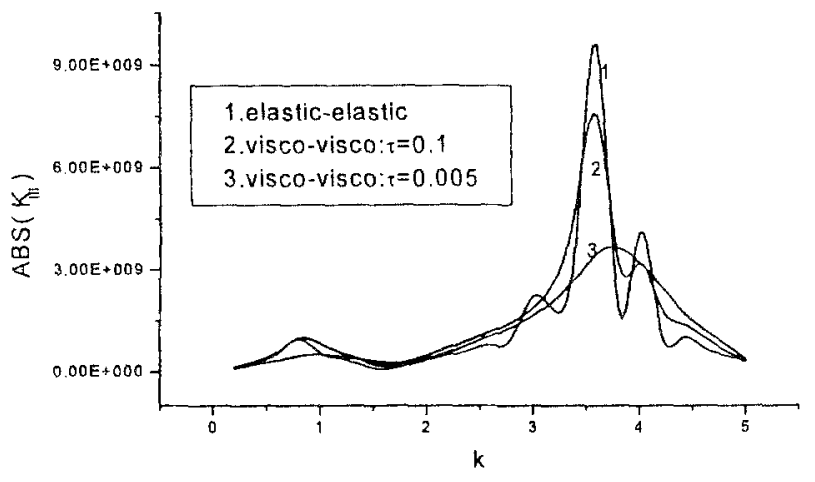

Fig. 5. Dynamic stress intensity factor $K_{\text {III }}$ for various relaxation times $\tau$

face crack are shown together for convenience of contrast. The effects of the relaxation time $\tau$ on the scattered stress field, in particular, on the peak value of the dynamic stress intensity factor $K_{\text {III }}$, are shown clearly.

From the numerical results shown in these figures, it can be learned that both of the crack open displacement and the stress intensity factor are different apparently between an elastic interface crack and a viscoelastic interface crack. The amplitude, distribution and mode of the crack open displacement are changed. In the case of normal incident, the crack open displacement $\Delta w$ is distributed symmetrically to the center of the crack, oblique incident makes the distribution of the crack open displacement along the crack face unsymmetric. Compared with that of the incident angle $\theta^{i}$, the effects of the wave number $k$ are much more complex. The effects of the wave number or incident frequency are connected with the amplitude, distribution and mode of the crack open displacement. The complexity caused by the incident frequency comes from the coupled effect of frequency and viscosity of the material. The stress intensity factor is dependent on the incident frequency and viscosity of the material. In a special frequency domain, the stress intensity factor shows resonance, and the peak value of resonance is controlled by the viscosity of the materials. The occurrence of the resonance can be explained as the constructive interference between the incident wave and the excited reflecting wave from the crack tip. Thus the appearence of resonance depends on the crack length and the material combinations.

In case of periodical harmonic load, two salient features of a viscoelastic material are phase shift between stress and strain and the energy dissipation by intrinsical friction. Strain takes place at the instant that stress is loaded in the elastic material. But the phase shifts existing in a viscoelastic material show that strain always lags behind stress. In order to describe the character of viscoelastic materials, the complex forms of stress, strain and elastic modulus are necessary. In general, the periodical harmonic stress $\tilde{\sigma}(\omega)$ and strain $\tilde{\varepsilon}(\omega)$ are related by the complex modulus $\tilde{E}(\omega)$

$\tilde{E}(\omega)=\frac{\tilde{\sigma}}{\tilde{\varepsilon}}=E e^{i \delta}=E_{1}(\omega)+i E_{2}(\omega)$,

where $\delta=\tan ^{-1}\left(E_{2}(\omega) / E_{1}(\omega)\right)$ is known as phase shift which represents the phase lag of strain relative to stress. Usually, the phase shift is a function of frequency. For a standard linear solid model, it is proved that phase shift tends to be infinitesimal at very low or very high frequency and prominent only in a limited frequency domain. Another remarkable feature is the energy dissipation that takes place in a viscoelastic material. The energy carried by the incident wave is transformed into kinetic energy and strain energy partly, and the rest is dissipated by intrinsic friction. For the spring-dashpot model of a linear viscoelastic material, 
it is assumed that energy is "stored" in the springs and "dissipated" in the dashpots. The mean energy flux $e$ in the incident wave can be expressed as

$\mathbf{e}=\langle\boldsymbol{\sigma} \cdot \dot{\mathbf{u}}\rangle$,

where $<>$ indicates averaging the product of the stress tensor and particle speed vector over one cycle. Then, the following energy balance equation holds:

$<\int_{V} D d V+\frac{\partial}{\partial t} \int_{V} W d V>=\int_{V}(\nabla \cdot \mathbf{e}) d V$.

For an inhomogeneous plane wave, the rate of dissipation by the medium per unit volume over one cycle can be expressed as

$<D>=-\nabla \cdot \boldsymbol{e}=2 \boldsymbol{e} \cdot \boldsymbol{P}_{I}^{i}$.

And the energy stored by the medium per unit volume, called energy density, can be expressed as

$<W>=\omega^{-1} \boldsymbol{e} \cdot \boldsymbol{P}_{\boldsymbol{R}}^{i}$.

It is clearly shown that the mean energy density depends on the component of energy flux normal to the lines of constant phase, while the mean rate of dissipation depends on the component of energy flux normal to the lines of constant amplitude. Because the energy carried by the incident wave is partially dissipated by intrinsic friction in a viscoelastic material, the stress intensity factor is damped down distinctively in contrast with that in an elastic material without energy loss. More energy is dissipated, more obviously the attenuation is. A dimensionless parameter defined to be the fractional energy loss per cycle is often used to express the attenuation and thus is called dissipation factor

$O^{-1}=\frac{<D>}{<W>\omega}=2 \frac{\boldsymbol{e} \cdot \boldsymbol{P}_{\boldsymbol{I}}^{i}}{\boldsymbol{e} \cdot \boldsymbol{P}_{R}^{i}}$

Obviously, the dissipation factor is related with frequency and complex modulus.

\section{Conclusion}

The scattering problem of a crack is important in theory and engineering. The corresponding problems for an elastic wave without energy loss are studied extensively, but rare for an inhomogeneous plane wave with energy loss. Obvious differences can be learned by comparing the numerical results of an elastic and viscoelastic wave. In this paper, emphasis is given to the crack opening displacement and the stress intensity factor which characterize the scattered near-field. The difference on amplitude, distribution and mode of the crack opening displacement results from the phase lag between strain and stress, and the attenuation of the stress intensity factor results mainly from the energy dissipation by intrinsic friction of viscoelastic materials. The effects of frequency of the incident wave on phase lag and energy dissipation play an important role. Because the complex modulus that is related closely to the phase lag and the dissipation factor that is related closely to the energy dissipation are both functions of the incident frequency, thus, the effects of frequency become complex and often couple together with that of the material. The coupled effects can account for the complex difference 
between crack open displacement of an elastic and viscoelastic interface crack. Energy dissipation varies with frequency either. At very low and high frequency, the energy dissipation can be neglected and is prominent only at a special frequency domain for a standard linear solid model, thus the attenuation of stress intensity becomes prominent in a special frequency domain. Relaxation time characterizes the viscosity of the material. The shorter the relaxation time is, the stronger the viscosity of material is and more prominent the attenuation of stress intensity is.

\section{Acknowledgement}

Research support provided by the Chinese Natural Sciences Foundation (No. 10032010 and No. 10172074) is gratefully acknowledged.

\section{References}

[1] Srivastava, K. N., Gupta, O. P., Palaiya, R. M.: Interaction of elastic waves in two bounded dissimilar elastic half-planes having Griffith crack at interface - I. Int. J. Fract. 14, 145-154 (1978).

[2] Srivastava, K. N., Palaiya, R. M., Gupta, O. P.: Interaction of longitudinal wave with penny-shaped crack at interface of two bounded dissimilar elastic bodies - II. Int. J. Fract. 15, $591-599$ (1979).

[3] Yang, H. J., Body, D. B.: Elastic wave scattering from an interface crack in a layered half-space. J. Appl. Mech. 52, 42-50 (1985).

[4] Bostrom, A.: Elastic wave scattering from an interface crack: antiplane strain. J. Appl. Mech. 54, $503-508$ (1987).

[5] Qu, J.: Interface crack loaded by a time-harmonic plane wave. Int. J. Solids Struct. 31, 329-345 (1994).

[6] Qu, J.: Scattering of plane waves from an interface crack. Int. J. Eng. Sci. 33, 179 - 194 (1995).

[7] Lockett, F. J.: The reflection and refraction of waves at an interface between viscoelastic materials. J. Mech. Phys. Solids 10, 53-63 (1962).

[8] Cooper, H. F., Reiss, E. L.: Reflection of plane viscoelastic waves from plane boundaries. J. Acoust. Soc. America 39, 1133-1138 (1966).

[9] Cooper, H. F.: Reflection and transmission of oblique plane waves at a plane interface between viscoelastic media. J. Acoust. Soc. America 42, 1064-1069 (1967).

[10] Schoenberg, M.: Transmission and reflection of plane waves at an elastic-viscoelastic interface. Geophys. J. R. Astr. Soc. 25, 35- 47 (1971).

[11] Borchert, R. D.: Reflection and refraction of type-II $S$ wave in elastic and anelastic media. Bull. Seis. Soc. America 67, 43-67 (1977).

[12] Muskhelishvili, I. N.: Singular integral equations. Groningen: Noordhoff 1953.

[13] Erdogan, F., Gupta, G. D., Cook, T. S.: Numerical solution of singular integral equations. In: Mechanics of fracture. V1. Methods of analysis and solutions of crack problems. (Sih, G. C., ed.). Groningen: Noordhoff.

[14] Kurtz, R. D., Farris, T. N., Sun, C. T.: The numerical solution of Cauchy singular integral equations with application to fracture. Int. J. Fract. 66, 139-154 (1994).

Authors' addresses: P. J. Wei, LNM, Institute of Mechanics, Chinese Academy of Science, Beijing 100080; Z. M. Zhang, Institute of Mechanics, Northern Jiaotong University, Beijing, 100044, China (E-mail: weipj@pku.edu.cn) 